\title{
Adaptive Phenotypic Plasticity of Siberian Elm in Response to Drought Stress: Increased Stomatal Pore Depth
}

\author{
Go Eun Park, ${ }^{1}$ Ki Woo Kim, ${ }^{2, \star}$ Don Koo Lee, ${ }^{1, \star}$ and Jung Oh Hyun ${ }^{3, \star}$ \\ ${ }^{1}$ Department of Forest Sciences, Seoul National University, Seoul 151-921, Korea \\ ${ }^{2}$ School of Ecology and Environmental System, Kyungpook National University, Sangju 741-711, Korea \\ ${ }^{3}$ Department of Forest Sciences, Institute of Future Environmental and Forest Resources, Seoul National University, \\ Seoul 151-921, Korea
}

\begin{abstract}
Leaf stomatal characteristics of Siberian elm (Ulmus pumila) were investigated by electron microscopy and white light scanning interferometry. On the basis of average annual precipitations, two types of tree specimens were collected from Korea, China, and Mongolia: (1) trees under normal environmental conditions and (2) trees under arid conditions. Field emission scanning electron microscopy revealed oval-shaped stomata on the lower surface, and they were ca. $20 \mu \mathrm{m}$ in width. In-lens secondary electron imaging showed differences in electron density and stomatal pore depth between the two types. According to the line profile analysis by white light scanning interferometry, stomata under arid conditions appeared to have higher levels of the stomatal pore depth than ones under normal conditions. Focused ion beam-field emission electron microscopy supported the increased stomatal pore depth with the increasing drought stress gradient. These results suggest that complementary microscopy can be employed to unravel the adaptive phenotypic plasticity of Siberian elm in response to drought stress.
\end{abstract}

Key words: drought, FIB, interferometry, stomata, Ulmus pumila

\section{INTRODUCTION}

Stoma (pl. stomata) is an opening or pore in the epidermis bordered by guard cells in plants (Evert, 2006). Stoma commonly includes both the pore and guard cells; stomatal complex refers to the stoma and any specialized epidermal cells adjacent to it (Mauseth, 1988; Carpenter, 2005). The main control of gas exchange and water movement in plants is provided by stomata (Cutler et al., 2008). Variations of stomatal structural characteristics are thought to affect gas exchange and the water pathways in the leaf (Roth-Nebelsick, 2007). The species' ability to respond and adapt to changing environments is critical for their survival and distribution.

Elms are deciduous trees belonging to the genus Ulmus in the northern hemisphere. Ulmus pumila L., commonly called Siberian elm, is a fast-growing and small- to mediumsized tree widely distributed in Korea, China, and Mongolia [Illinois Nature Preserves Commission (INPC), 1990]. The trees have been preferentially planted in Mongolia as main broadleaved species for the rehabilitation of arid areas (Shi et al., 2004; Valladares \& Sánches-Gómez, 2006). Investigating stomatal characteristics of species tolerant to drought stress would provide a better understanding of tree adaptations in harsh environment.

Although field emission scanning electron microscopy (FESEM) provides a high-resolution image of stomatal com-

() MICROSCOPY SOCIETY OF AMERICA 2013

*Corresponding authors. E-mail: kiwoo@knu.ac.kr; leedk@snu.ac.kr; junghyun@ snu.ac.kr plexes, accurate measurement of the $z$-axis dimension is limited because of the imaging principles. White light scanning interferometry (WLSI) has been used to provide threedimensional metrology of plant leaves (Kim et al., 2010). In addition, focused ion beam (FIB) systems have been increasingly used for biological materials, as well as metals and semiconductors (Hou \& Yao, 2007). Combining the FIB's precise machining abilities with the FESEM's high-resolution imaging abilities, the two-beam system (FIB-FESEM) leads to novel applications (Yao, 2007). Here we report the morphological response of Siberian elm under different levels of drought stress, and provide an experimental evidence on the quantitative analysis of the vertical profiles of leaf stomata.

\section{Materials and Methods}

\section{Plant Materials}

Leaves of $U$. pumila were collected from ca. 60-year-old field-grown trees in four sites: (i) Jeongseon, Korea (JS), (ii) Qingyuan, China (QI), (iii) Kokunhang, Mongolia (KK), and (iv) Wulanaodu, China (WU). The trees were subjected to different levels of water supply conditions during their growing seasons over the past decades. On the basis of average annual precipitations, they were classified into two categories: (i) normal conditions [JS $(1,316 \mathrm{~mm})$ and QI $(634 \mathrm{~mm})]$ and (ii) arid conditions [KK $(235 \mathrm{~mm})$ and WU $(262 \mathrm{~mm})]$. Leaves were taken from the middle part of the crown that was exposed to full sunlight throughout the day. 

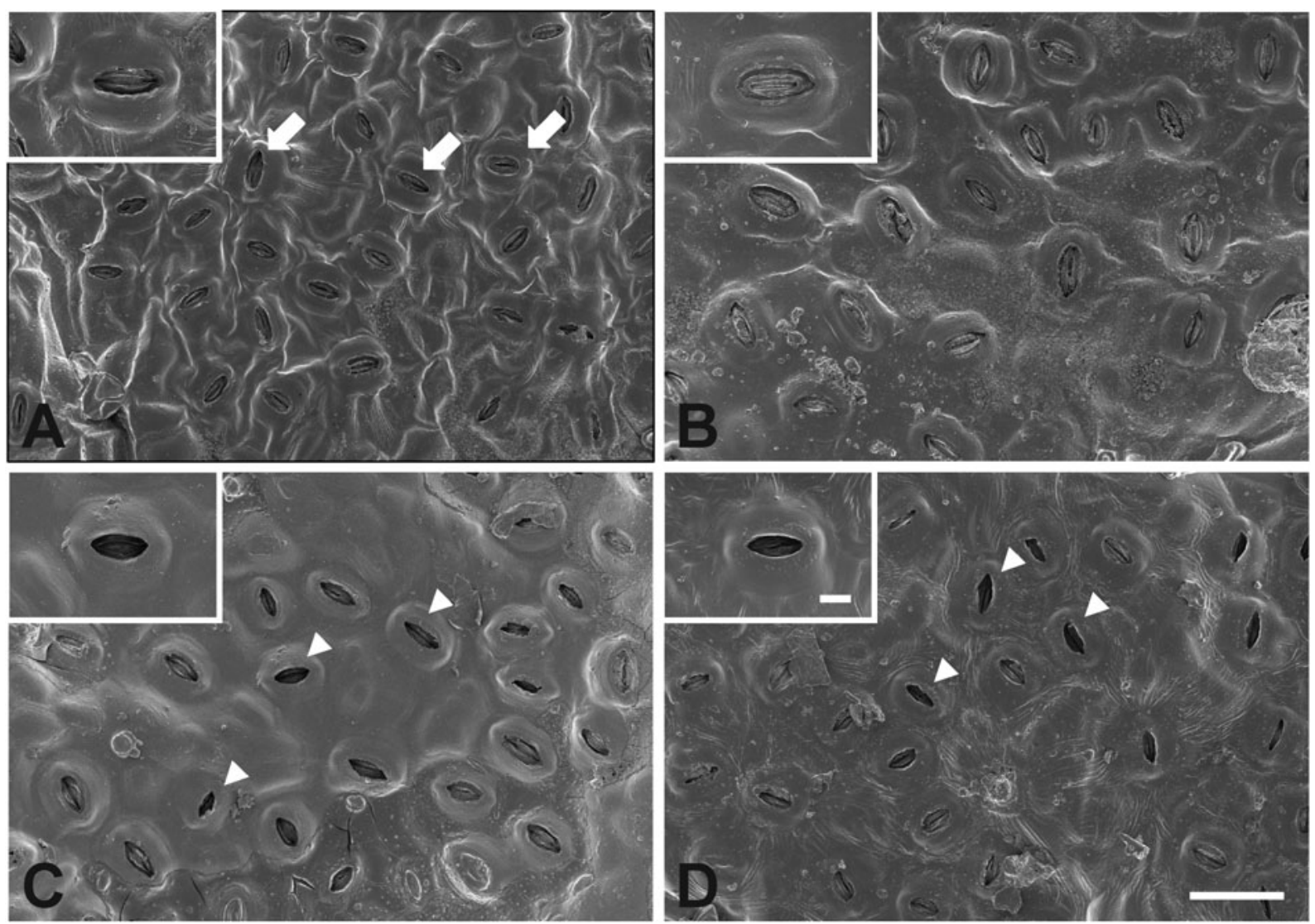

Figure 1. Field emission scanning electron microscopy of leaves of Ulmus pumila: (A) leaves from Jeongseon (arrows indicate stomata on the leaf surface); (B) leaves from Qingyuan; (C) leaves from Kokunhang; (D) leaves from Wulanaodu. The stomatal pores under arid conditions [arrowheads in (C) and (D)] appeared to be more deeply located relative to the epidermis than those under normal conditions. Scale bar is $20 \mu \mathrm{m}$. (Rectangles show higher magnifications of stomata. Scale bar is $5 \mu \mathrm{m}$.)

\section{FESEM}

Leaf fragments were mounted on a stub and sputter-coated with platinum $(\mathrm{Pt})$ using a coater and examined by FESEM (Supra 55VP; Carl Zeiss, Oberkochen, Germany) at 2 kV. An annular-type in-lens detector was used to acquire secondary electron signals. Stomatal dimensions were measured using a software package.

\section{WLSI}

Optical scanning interferometry was performed for the quantitative analysis of stomatal complexes (NanoViewE1000; NanoSystem, Daejeon, Korea). Line profile analysis was conducted to measure the stomatal pore depth (from epidermis to pore) of 30 stomatal complexes (Kim et al., 2011). The mean value of stomatal pore depth was compared among sites by Duncan's multiple range test.

\section{FIB-FESEM}

The Pt-coated leaf specimens were mounted on FIB-FESEM (Auriga; Carl Zeiss). Coarse cross-sections of stomatal complexes were made with a $10-\mathrm{nA}$ ion beam current and fine-polished with a 50-pA ion beam current. An in-column energy-selective backscattered electron (EsB) detector provided the exposed surface image (Kim \& Jaksch, 2009). The stomatal pore depth was measured from 20 stomatal complexes and analyzed as described above.

\section{Results}

\section{FESEM}

Stomata were present only on the lower leaf surface, ovalshaped, and ca. $20 \mu \mathrm{m}$ in width (Fig. 1). No significant differences in stomatal morphology were observed between leaves under normal conditions (Figs. 1a, 1b). The electrondense stomatal pores commonly observed on the leaf surface under arid conditions (Figs. 1c, 1d) appeared to be more deeply located relative to the epidermis than those under normal conditions.

\section{WLSI}

Surface topography of leaves was revealed in scan areas (Fig. 2a). Surface fluctuations in height profiles were evident by the color scale (Fig. 2b), and the pore regions in the center were demarcated from the outer stomatal chamber. An arbitrary line profile analysis allowed for the measurement of the stomatal pore depth (Fig. 2c). The leaves under arid conditions had a higher stomatal pore depth (ca. $3.0 \mu \mathrm{m})$ than those under normal conditions (ca. $1.8 \mu \mathrm{m}$ ). 

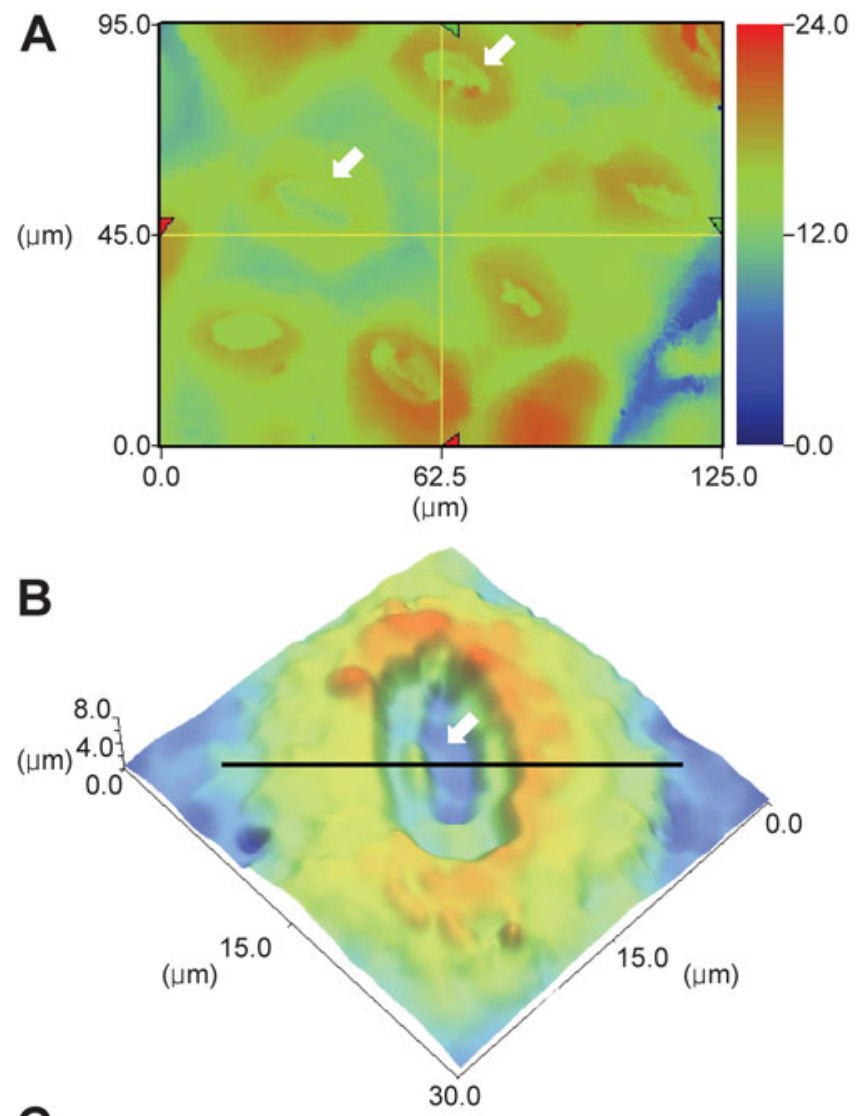

C

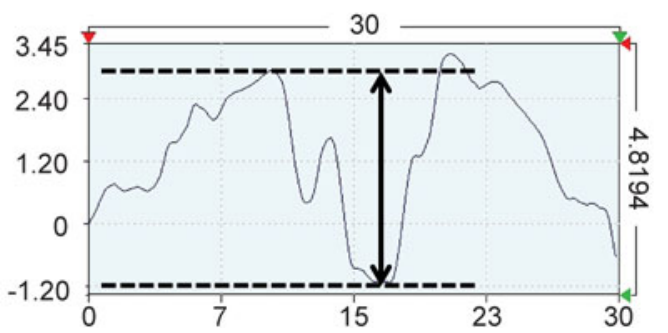

Figure 2. White light scanning interferometry of stomata. A: Twodimensional surface plot; arrows indicate stomata. B: Threedimensional surface plot. A line represents the arbitrary line profile analysis of the stoma. An arrow indicates the pore of the stomatal complex. C: $X$-direction line profile of the stoma in (B). The stomatal pore depth was measured from the image.

\section{FIB-FESEM}

Based on the vertical planes of stomatal complexes, it was obvious that the depth difference between the leaf epidermis and pore increased under arid conditions (Fig. 3). Significantly different levels of stomatal pore depth were found between leaves from the normal conditions (ca. $3 \mu \mathrm{m}$ ) and arid conditions (ca. $5 \mu \mathrm{m}$ ) (Fig. 4). No significant differences were found in stomatal pore depth within the same groups $(p=0.05)$.

\section{DiscusSION}

This study provided an experimental evidence for the adaptive response of leaf stomata to drought stress in Siberian elm. It was found that naturally reducing water supply in
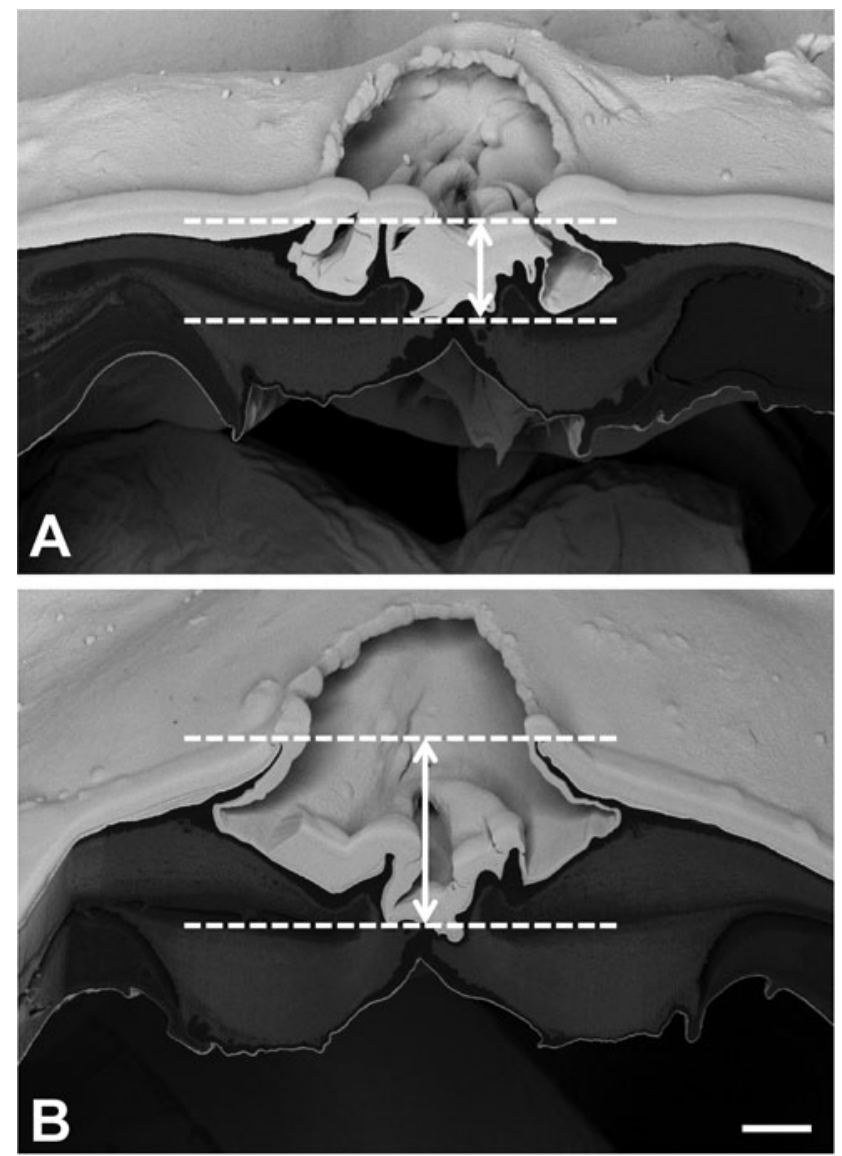

Figure 3. Focused ion beam-field emission scanning electron microscopy of stomata: (A) stoma from Jeongseon; (B) stoma from Kokunhang. Arrows indicate the stomatal pore depth on the vertical planes of stomatal complexes. The depth difference between the leaf epidermis and pore increased under arid conditions. Scale bar is $2 \mu \mathrm{m}$.

the forest stand caused an alteration of stomatal architecture of Siberian elm. The most striking differences between stomata were found in the pore position of stomatal complexes. Stomata under arid conditions were mainly characterized by the more sunken stomatal pores. Qualitative features of sunken stomatal pores were visualized by FESEM where a coaxial in-lens imaging provided a clue to further address the differences between stomata from the leaves of the environments.

In addition, quantitative aspects of stomatal architecture were analyzed by WLSI and FIB-FESEM. Although Pt-coated leaves for FESEM were scanned for WLSI in this study, even the light reflection from uncoated raw leaf materials was sufficient to generate three-dimensional surface plot (Kim et al., 2011). The measurements of stomatal pore depth by WLSI may reflect the drought stress gradients.

The FIB-FESEM was effective to expose the vertical profiles of leaf surface with indiscernible beam damage. EsB imaging was sufficient to resolve the vertical profiles of leaf surface, based on the compositional contrast. The increase in stomatal pore depth measured by FIB-FESEM was in agreement with the drought stress gradient of the sites. 


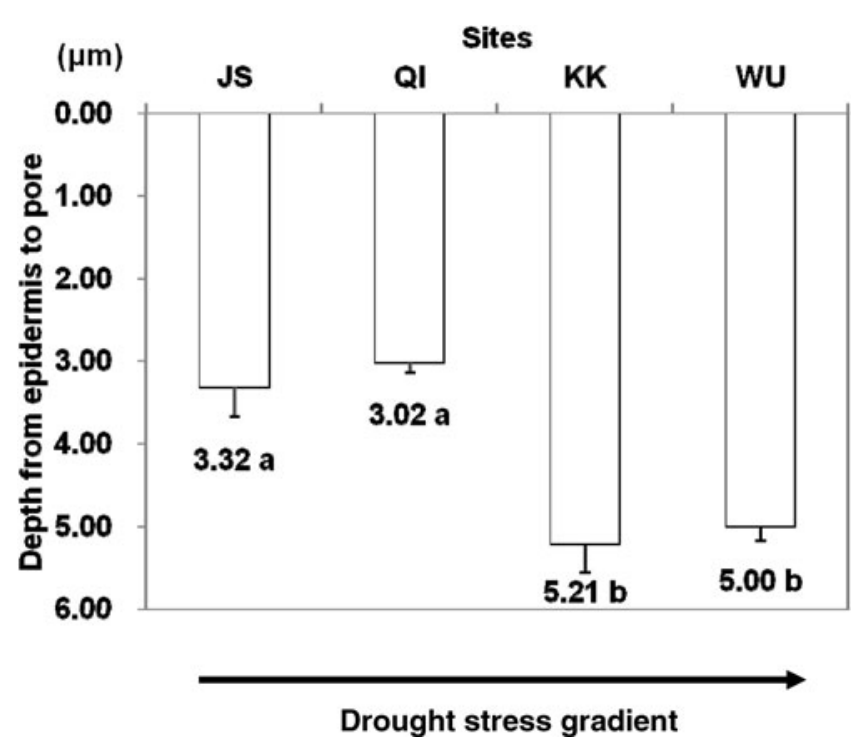

Figure 4. Stomatal pore depth. Significantly different levels of depth were found between leaves under different environments. JS, Jeongseon; QI, Qingyuan; KK, Kokunhang; WU, Wulanaodu.

Differences in stomatal pore depth between WLSI and FIBFESEM might be attributed to several factors including specimen orientation and spatial resolution of beam source.

Plants exposed to continued arid conditions tend to improve water use efficiency by minimizing water loss. It is natural that stomata under arid conditions have sunken stomatal pores (Cutler et al., 2008). Such architectural characteristics, owing to their boundary layer conductance, may contribute to the shielding of stomata from the drying winds in arid areas, and keep the stomata less vulnerable to water loss than other stomata types. To our knowledge, this is the first report on the use of complementary microscopy to unravel the stomatal response to drought stress in plants.

In summary, our findings indicate that stomatal architecture is plastic to drought stress in Siberian elm. The stomatal pore positions can be altered to maintain the water use efficiency to survive under adverse conditions. Increased stomatal pore depth is regarded as a morphological response to drought stress, which could be quantified by microscopy. Elucidation of triggering factors and thresholds of environmental pressure for stomatal modifications will enhance our understanding of the nature of tree responses to drought stress.

\section{ACKNOWLEDGMENT}

This study was carried out with the support of "Key Joint Research Program" provided by the National Research Foundation of Korea.

\section{REFERENCES}

Carpenter, K.J. (2005). Stomatal architecture and evolution in basal angiosperms. Amer J Bot 92, 1595-1615.

Cutler, D.F., Botha, T. \& Stevenson, D.W. (2008). Plant Anatomy. An Applied Approach. Malden, MA: Blackwell Publishing.

Evert, R.F. (2006). Esau's Plant Anatomy: Meristems, Cells, and Tissues of the Plant Body-Their Structure, Function, and Development, 3rd ed. Hoboken, NJ: John and Wiley Sons.

Hou, K. \& YAO, N. (2007). Applications for biological materials. In Focused Ion Beam Systems. Basics and Applications, Yao, N. (Ed.), pp. 337-354. Cambridge, UK: Cambridge University Press.

Illinois Nature Preserves Commission (INPC) (1990). Vegetation Management Guidelines: Siberian elm (Ulmus pumila L.), vol. 1, pp. 1-4. Springfield, IL: INPC.

KIM, K.W. \& JAKsch, H. (2009). Compositional contrast of uncoated fungal spores and unstained section-face by low-loss backscattered electron imaging. Micron 40, 724-729.

Kim, K.W., Kim, D.H., Han, S.H., Lee, J.C. \& Kim, P.G. (2010). Three-dimensional surface topography of the needle stomatal complexes of Pinus rigida and its hybrid species by complementary microscopy. Micron 41, 571-576.

Kim, K.W., Lee, I.J., Kim, C.S., Lee, D.K. \& PARK, E.W. (2011). Micromorphology of epicuticular waxes and epistomatal chambers of pine species by electron microscopy and white light scanning interferometry. Microsc Microanal 17, 118-124.

Mauseth, J.D. (1988). Plant Anatomy. Caldwell, NJ: The Blackburn Press.

Roth-Nebelsick, A. (2007). Computer-based studies of diffusion through stomata of different architecture. Ann Bot 100, 23-32.

ShI, L., Zhang, Z.J., Zhang, C.Y. \& Zhang, J.Z. (2004). Effects of sand burial on survival, growth, gas exchange and biomass allocation of Ulmus pumila seedlings in the Hunshandak Sandland, China. Ann Bot 94, 553-560.

Valladares, F. \& SÁnches-Gómez, D. (2006). Ecophysiological traits associated with drought in mediterranean tree seedlings: Individual responses versus interspecific trends in eleven species. Plant Biol 8, 688-697.

YAO, N. (2007). Introduction to the focused ion beam system. In Focused Ion Beam Systems. Basics and Applications, Yao, N. (Ed.), pp. 1-30. Cambridge, UK: Cambridge University Press. 